\title{
NAV-Landmarks: Deployable 3D Infrastructures to Enable CubeSats N avigation Near Asteroids
}

\author{
Marco Zaccaria Di Fraia \\ Centre for Electronic Warfare \\ Information and Cyber \\ Cranfield University, The \\ Defence Academy Of The UK \\ Shrivenham, SN6 8LA, UK \\ marco.di-fraia@cranfield.ac.uk
}

\author{
Lounis Chermak \\ Centre for Electronic Warfare \\ Information and Cyber \\ Cranfield University, The \\ Defence Academy Of The UK \\ Shrivenham, SN6 8LA, UK \\ 1.chermak@cranfield.ac.uk
}

\author{
Joan-Pau Cuartielles \\ Space Research Group, Centre of \\ Autonomous and Cyber-Physical \\ Systems \\ Cranfield University \\ MK43 0AL, Bedfordshire, UK \\ jp.sanchez@cranfield.ac.uk
}

\author{
Leonard Felicetti \\ Space Research Group, Centre of \\ Autonomous and Cyber-Physical \\ Systems \\ Cranfield University \\ MK43 0AL, Bedfordshire, UK \\ leonard.felicetti@cranfield.ac.uk
}

\author{
Antonio Fulvio Scannapieco \\ Centre for Electronic Warfare \\ Information and Cyber \\ Cranfield University, The \\ Defence Academy Of The UK \\ Shrivenham, SN6 8LA, UK \\ a.scannapieco@cranfield.ac.uk
}

\begin{abstract}
Autonomous operations in the proximity of Near Earth Objects (NEO) are perhaps the most challenging and demanding type of mission operation currently being considered. The exceptional variability of geometric and illumination conditions, the scarcity of large scale surface features and the strong perturbations in their proximity require incredibly robust systems to be handled. Robustness is usually introduced by either increasing the number and/or the complexity of on-board sensors, or by employing algorithms capable of handling uncertainties, often computationally heavy. While for a large satellite this would be predominantly an economic issue, for small satellites these constraints might push the ability to accomplish challenging missions beyond the realm of technical possibility. The scope of this paper is to present an active approach that allows small satellites deployed by a mothership to perform robust navigation using only a monocular visible camera. In particular, the introduction of Non-cooperative Artificial Visual landmarks (NAVLandmarks) on the surface of the target object is proposed to augment the capabilities of small satellites. These external elements can be effectively regarded as an infrastructure forming an extension of the landing system. The quantitative efficiency estimation of this approach will be performed by comparing the outputs of a visual odometry algorithm, which operates on sequences of images representing ballistic descents around a small non-rotating asteroid. These sequences of virtual images will be obtained through the integration of two simulated models, both based on the Apollo asteroid 101955 Bennu. The first is a dynamical model, describing the landing trajectory, realized by integrating over time the gravitational potential around a three-axis ellipsoid. The second model is visual, generated by introducing in Unreal Engine 4 a CAD model of the asteroid (with a resolution of $75 \mathrm{~cm}$ ) and scattering on its surface a number $N$ of cubes with side length $L$. The effect of both $N$ and $L$ on the navigation accuracy will be reported. While defining an optimal shape for the NAV-Landmarks is out of the scope of this paper, prescriptions about the beacons geometry will be provided. In particular, in this work the objects will be represented as high-visibility cubes. This shape satisfies, albeit in a non-optimal way, most of the design goals.
\end{abstract}

\section{TABLE OF Contents}

1. INTRODUCTION ........................................................1

2. STATE OF THE ART …..................................................2

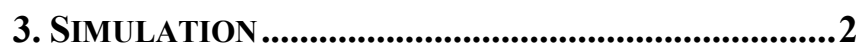

4. LANDMARK DESIGN.................................................6

5. RESULTS ......................................................................

6. CONCLUSIONS .............................................................11

APPENDICES ..................................................................11

A. DEFINING THE LANDMARKS POSITION................11

B. SELECTING AN ERROR METRIC ............................12

ACKNOWLEDGEMENTS .........................................12

REFERENCES ........................................................12

BIOGRAPHY ..............................................................

\section{INTRODUCTION}

Interplanetary CubeSats are unquestionably still an emerging technological field: at the time of writing, less than a year has passed from the first, and for now only, mission of this type, Mars Cube One (MarCO) [1].

A CubeSat is a small satellite designed complying with a particular standard, defined by Bob Twiggs and Jordi PuigSuari in 1999 [2]. The design prescription requires these spacecraft to be a small collection of basic "Units" (1U), defined as cubes with sides measuring $10.0 \mathrm{~cm}$ and a mass smaller than $3.00 \mathrm{lbs}(1.33 \mathrm{~kg})$. Typical architectures are $1 \mathrm{U}, 2 \mathrm{U}, 3 \mathrm{U}, 6 \mathrm{U}$ and $12 \mathrm{U}$. This restricted volume limits the amount of resources available on board, substantially constraining the level of autonomy achievable by these small satellites. While this shortcoming might not be a problem when orbiting in proximity to the Earth, since human controllers or other forms of navigational support might compensate for it, it becomes critical for deep space missions. 
Whereas it might be difficult for a CubeSat to carry out a complex mission by itself, a winning paradigm to employ these spacecraft might lie in integrating their capabilities with either other satellites or some form of infrastructure. This could mean either extending the capabilities of CubeSats through cooperation or interaction with other entities, or further augmenting more complex probes through small satellites employed as external subsystems. As an example of the latter, the two $6 \mathrm{U}$ orbital modules making up the MarCO mission, MarCO-A and MarCO-B, were used as an additional detached communication subsystem for the InSight lander, acting as beyond-LOS orbital relays during its EDL phase [3].

This work explores this idea of CubeSats operating as part of a hybrid distributed system, encompassing the landing probe and of a set of passive beacons serving as navigational aids. In particular, the hypothesis under test in this work is that the addition of artificial features to the surface of the landing target enhances the accuracy of optical estimations at a medium distance.

While this argument might sound intuitively true, it is necessary to verify it. Moreover, in order to justify the nonnegligible impact of the deployable markers on the critical mass budget, their effectiveness must be objectively proved and quantified. This paper will do so, focusing on a particular target, namely small asteroids. This because their reduced size allows for the implementation of a whole-target coverage dispersion configuration, in addition to the commonly studied pseudo-clustering in the expected landing area.

A whole-target coverage approach can be regarded as an inverse-GPS architecture, with an orbiting receiver and a constellation-like infrastructure on the ground. In particular, the studied scenario contemplates the ballistic deployment of a number $n$ of small markers by a mothercraft, implemented before the deployment of a CubeSat lander. While the challenges for mission design are not explored in this paper, it is assumed that the markers are extendable devices requiring little mass and volume to be transported.

After discussing the state of the art concerning fiducial markers for asteroids proximity operations in Section II, every aspect of the simulation design process will be thoroughly described and explained in Section III. Section IV briefly introduces considerations regarding the possible design constraints of the landmarks, while, ultimately, Section V defines a specific test case and presents the effect of the landmarks on a visual navigation algorithm.

\section{State OF THE ART}

The key to far-reaching, cost-effective space operations lies in warehousing [4]. To establish a warehouse means to structure a repository of modular, specialised, solutions which can easily be integrated and deployed. The usual approach to assemble this collection is to develop and collect sensors and algorithms. What is attempted in this work is to investigate a paradigm shift. Namely, focusing the warehousing efforts on developing a set of infrastructures used to structure the environments as default operating spaces. In one of its primitive iterations, this architecture would be realized through reference guideposts scattered on the target surface of interest: these are the NAV-Landmarks found in the title.

Using artificial markers as an aid while performing visual controlled tasks is a rather common strategy for an endless range of operations, either manned or autonomous. Indeed, typical applications may span from on-orbit proximity operations [5] to UAVs landing [6], from navigation of terrestrial robots [7] to underwater applications [8]. The function of fiducials is to provide consistent, standardized information and cues, used to augment the environment or a target into a cooperative form. A cooperative entity provides to the observing subsystem operative information that need little or no processing to be used.

These features can be either added while designing the target (for example, for servicing) or deployed at a later stage. This means that the operative methodology can be calibrated on operating using principally these elements, known a priori.

This approach, however, seems to have been mostly neglected by the space scholars and engineers for applications beyond Earth's orbit. There, coherently with the rest of the computer vision community, the research trends appears to be oriented towards highly centralized, marker-less solutions (e.g. [9]) or towards high environmental specialization (e.g. [10]). The constraints over volumetric and mass budgets are rarely as severe as in the space domain, and transporting markers would negatively impact them, potentially severely.

Ultimately, we mainly expect three factors to influence the realization of this scheme. These are the mass-to-intelligence costs ratio, the trends concerning space exploration strategies, and the development of expandable structures [11], [12].

However, despite the listed constraints, there have been two instances in the last few years where this technology has been employed in a space missions. Namely, instances of this technology have already been used, on the JAXA probe Hayabusa [13], and its successor, Hayabusa2 [14]. Both these probes carried on board, respectively, three and five highly reflective orbs, to enable close-range navigation for a pinpoint autonomous landings. More details about the objects themselves will be provided in Section V. To further exploit the reflectivity of the orbs during camera observations, both probes were equipped with a flashlight (FLA instrument) to highlight the markers on the surface. The programmed markers release altitude had been set to roughly 30 meters in both cases [14], [15]. However, the deployment was realized at 40 meters for Hayabusa [15] and reported to happen around 10 meters in subsequent Hayabusa2 sources [16]. The features injection strategy envisions them deployed not all at once, but iteratively, with the already deployed markers used to increase the deployment accuracy of the subsequent ones, ultimately forming a pseudo-cluster around the desired landing area. The augmentation provided by these objects can then be integrated in a wide array of close-range Guidance and Navigation strategies [15], [17].

\section{Simulation}

The overall navigation analysis is going to be performed on a visuodynamical simulation. That is, a simulation coupling optical and dynamical effects, constructed overlapping and integrating two models generated through two different approximations of the same object. Specifically, both approximations are constructed from a non-rotating $(\omega=0)$ object having density and volume identical to the asteroid 101955 Bennu, towards which the CubeSat performs a ballistic descent. However, whereas the camera output simulation is built around an object reconstructed from the real Bennu, the descent dynamics propagated in the visual 
model is generated by approximating the asteroid as a threeaxis ellipsoid with uniform density.

\section{A. Orbital Mechanics}

Let us assume that the gravity field of Bennu can be wellapproximated by the one induced by a uniform mass distribution shaped as a tri-axial ellipsoid. The principal axes of the ellipsoid are going to be considered aligned with the standard basis defining the Inertial Reference Frame, and indicated, respectively, as $R_{x}, R_{y}$ and $R_{z}$. Using them, every point lying on the surface of the ellipsoid can be described by the equation

$$
\frac{x^{2}}{R_{x}^{2}}+\frac{y^{2}}{R_{y}^{2}}+\frac{z^{2}}{R_{z}^{2}}=1
$$

A first naïve choice for these axial extensions could be represented by using the real radii of the spinning-top-shaped Bennu. However, this would lead to a grossly overestimated volume, and given that when using a constant density model ( $\rho=$ const) the mass $M$ varies linearly with the volume $V$

$$
M=\rho V
$$

Ultimately, through those numbers an overestimation of the mass would have been obtained. The logical solution to this seem, consequently, to employ a volume-preserving equivalence. Such a reduction to an isovolumetric ellipsoid can be operated by transforming the object into its DEEVE, Dynamically Equivalent Equal Volume Ellipsoid, which adds also the useful constraint of dynamical (i.e. inertial) equivalence. Values for the $R_{x_{i}}^{D E E V E}(i=1,2,3)$ of Bennu can be found in [18]. Some updated, post-rendezvous, data were recently presented in Lauretta et al. [19]. While among those data it is not possible to find updated figures for the DEEVE axes, it is possible to observe how the values measured on-site are reasonably close to the ones presented in [18]. Therefore, we feel confident that even by using preencounter values the output model would be satisfactorily realistic.

As a consequence of the $\omega=0$ hypothesis, completing the set of parameters used to define the orbital mechanics model requires only to define the density. The average bulk density can easily be retrieved from [19]. Through equation (3) it is possible to observe that using a DEEVE constrained by old volumes data, and using an updated value of $\rho$ leads the mass of the simulated object to be to adequately close to the real one. Indeed, the difference between the model's mass and the real one is $<1 \%$ of the actual mass. In conclusion, the design choices presented lead all the inertial properties to be adequately approximated in the virtual model. Therefore, the asteroid employed within the Orbital Mechanics simulation will have the following characteristics, listed here for reference:

$$
\left\{\begin{array}{c}
\omega=0 \\
\rho=1190\left[\mathrm{kgm}^{-3}\right] \\
R_{x}=259[\mathrm{~m}] \\
R_{y}=251[\mathrm{~m}] \\
R_{z}=234[\mathrm{~m}]
\end{array}\right.
$$

The resulting ellipsoid can be observed in Fig. 1, textured with a map of Bennu's surface retrieved from [20] for graphic

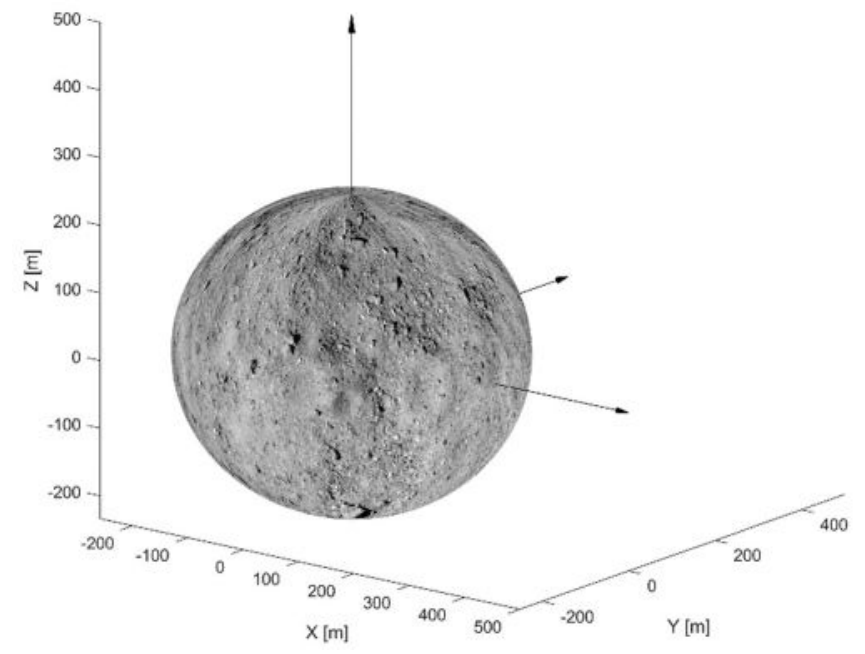

Fig. 1. The ellipsoid to which Bennu has been reduced

purposes. Fig. 2 and Fig. 3 show the way in which this object deviates from the high resolution CAD model.

This set of information is sufficient to model the gravity potential emanating from this volume, which can be used to propagate the probe trajectory from an initial condition. In particular, the descent path will be generated by selecting the desired landing configuration, and propagating it back to a specific state in space. This state can either be the deployment condition or a generic position along the trajectory. The parameter controlling the selection of this furthest estimated state is the amount of simulated falling time. The backpropagation operation is executed by iteratively solving a set of ODEs (using the MATLAB function ode45) defining the state evolution. Being the attitude of the platform constant, the state $\underline{X}$ at a generic instant $t$ will be defined only by the position and the velocity of the platform. This means that $\underline{\dot{X}}$ contains the probe's linear velocities and accelerations. The potential, function of the distance from the center of the ellipsoid, $\underline{\boldsymbol{r}}$, will be characterized as an integral and is expressed as reported by Scheeres [21] as

$$
U(\underline{\boldsymbol{r}})=-\frac{3 \mu}{4} \int_{\lambda(\underline{\boldsymbol{r}})}^{\infty} \varphi(\underline{\boldsymbol{r}}, u) \frac{d u}{\Delta(u)}
$$

Where $\varphi(\boldsymbol{r}, u)$ is a rational function and $\lambda(\boldsymbol{r})$ its largest real root in $u$. The form of the first-order partial derivatives $U_{x}, U_{y}$ and $U_{z}$ descending from this potential, as reported in [21], allows their estimation to be performed using Carlson's duplication method [22]. The acceleration induced on the platform along any of the axes by the rotating, gravitationally attractive body is the sum of the partial derivative of the potential along that axis and additional rotational terms.

Since the constraints imposed in this scenario lead to rotational terms identically equal to zero, ultimately the linear accelerations map, axis by axis, the partial derivatives of the gravitational potential.

Within the scope of this paper, the propagated landing state, let it be the vector $\underline{\boldsymbol{X}}^{L}$, can be unambiguously defined using six parameters. In particular, these are the three components of the landing velocity, $\underline{V}^{L}$, and the three components of the landing position, $\underline{S}^{L}$. In short, $\underline{X}^{L}=$ $\left(\underline{\boldsymbol{S}}^{L}, \underline{\boldsymbol{V}}^{L}\right)=\left(S_{x}^{L}, S_{y}^{L}, S_{z}^{L}, V_{\boldsymbol{x}}^{L}, V_{\boldsymbol{y}}^{L}, V_{z}^{L}\right)$. In particular, a vertical landing along the Equator will be studied. 


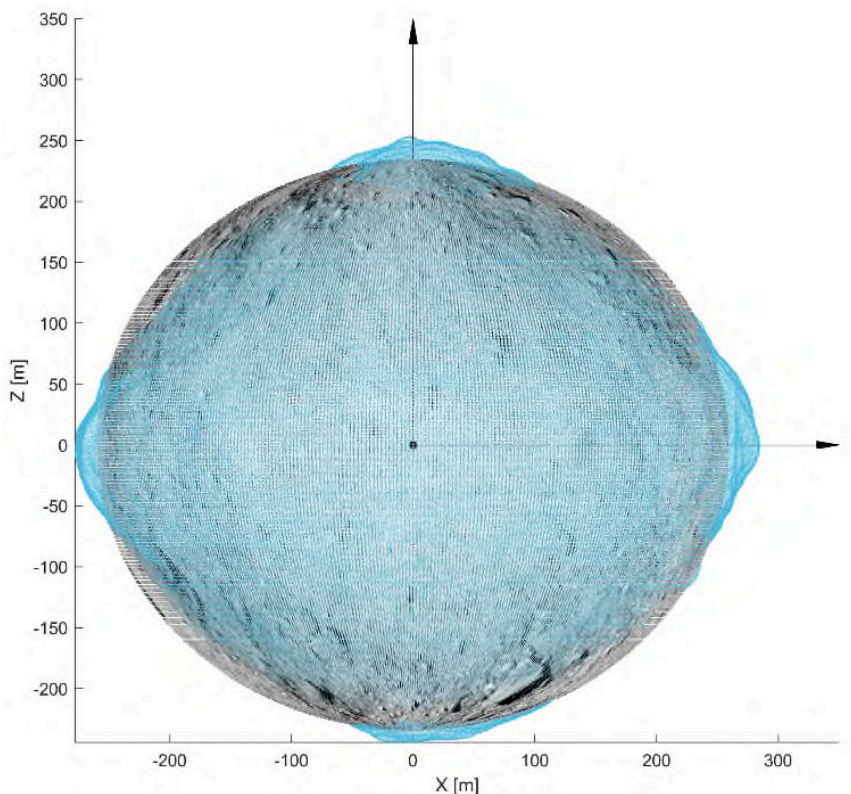

Fig. 2. The discrepancy between the DEEVE ellipsoid and the CAD model in the $\mathrm{X}-\mathrm{Z}$ plane (MATLAB, right handed frame)

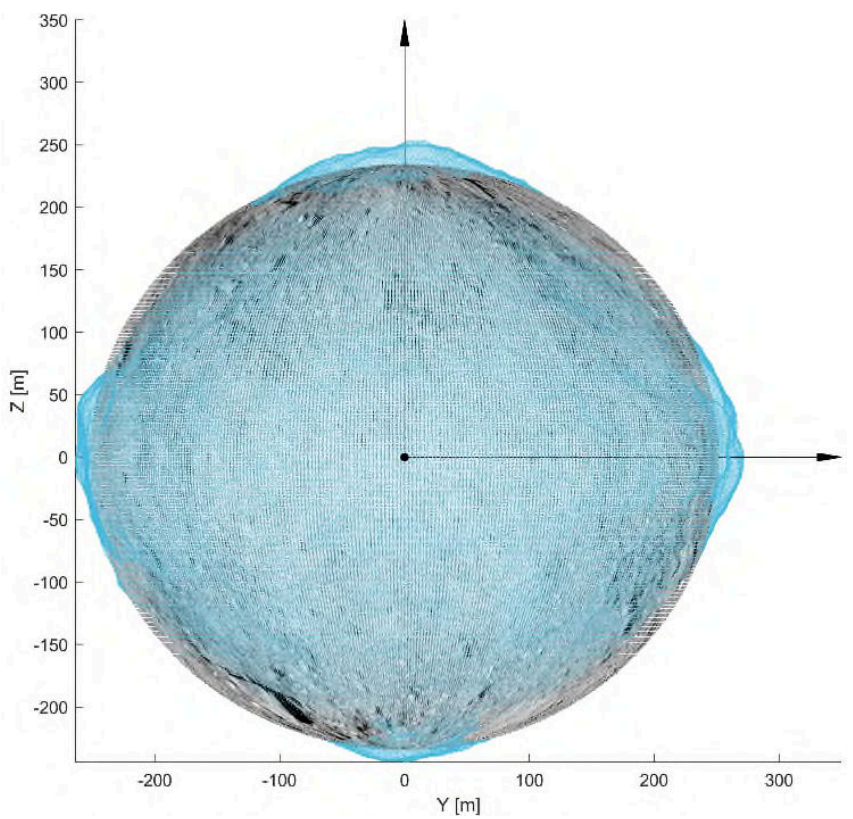

Fig. 3. The discrepancy between the DEEVE ellipsoid and the CAD model in the Y-Z plane (MATLAB, right handed frame)

The magnitude of $\underline{V}^{L}$ has to be constrained so as to allow the probe not to bounce back into space after the touchdown. This upper bound will be defined Critical Landing Velocity, $\underline{V}_{c}^{L}$. Namely, this is the highest velocity with which the probe can reach any point on the surface without bouncing back into deep space after the impact. This means that for $\left\|\underline{\boldsymbol{V}}_{L}\right\|<\left\|\underline{\boldsymbol{V}}_{c}^{L}\right\|$, the probe is ideally bound to be on the surface of the asteroid after the touchdown. Such a cap, coupled with the low gravity of the target will imply a consistently slow falling dynamics, with direct consequences on the navigation design process.

Let $\epsilon$ be the local normal Coefficient of Restitution, a parameter correlated to the kinetic energy lost in a collision event, defined as

$$
\epsilon=\frac{V^{a}}{V^{L}}
$$

By definition, $\epsilon$ is the ratio of the components along the local vertical of the relative velocities of two masses, before and after their collision. . It is possible to consider only the velocity of the probe because the asteroid is non-rotational and negligibly perturbed by the probe impact, and the landing orthogonal to the surface. Therefore $V^{L}$ is reduced to the probe speed before the impact and $V^{a}$ to the probe speed after the impact.

Not taking into account very special cases, $\epsilon$ is bound to be such that $0 \leq \epsilon \leq 1$, with $\epsilon=1$ representing a perfectly elastic impact, with no energy dissipation. Now, let $\epsilon_{\text {crit }}$ be the highest value of $\epsilon$ that can be found on the surface. This value is deemed critical because a sufficiently high $V^{a}$ not suppressed by an adequate $\epsilon$ might lead to losing the probe forever.

$V_{c}^{L}$ is obtained by forcing the condition that the velocity after the impact has to be less than the lowest value of the escape velocity, $V_{e}^{\min }$. In this way, the first, most energetic, bounce away from the surface does not possess enough kinetic energy to climb outside the local gravity well.

The escape velocity is defined as

$$
V_{e}(m, \underline{\boldsymbol{r}})=\sqrt{\frac{2 G m}{R}}=\sqrt{\frac{2 G m}{\sqrt{r_{x}^{2}+r_{y}^{2}+r_{z}^{2}}}}
$$

Since the gravitational constant is an universal constant, and the mass is a property of the whole object, $V_{e}^{\min }$, is realized in the point of the object farthest from its center of gravity, let it be $R_{M}$. Therefore

$$
V^{a}<V_{e}^{\min }=\sqrt{\frac{2 G m}{R_{M}}}
$$

And being that

$$
\epsilon=\frac{V^{a}}{V^{L}} \Leftrightarrow \epsilon V^{L}=V^{a}
$$

Ultimately this condition translates to

$$
\epsilon V^{L}<\sqrt{\frac{2 G m}{R_{M}}}
$$

Thus implying

$$
V_{c}^{L}=\sqrt{\frac{2 G m}{\epsilon_{c r i t}^{2} R_{M}}}
$$

\section{B. Virtual Reality Development Toolbox}

The first step in modelling a graphical virtual reality is the selection of the set of tools used to construct the simulation. For this work three equally plausible development alternatives were evaluated: PANGU [23], the Simulink 3D Animation toolbox [24] and Unreal Engine 4. The choice, ultimately, fell on Unreal Engine 4 [25] (specifically, UE4.22.3). At first this selection might appear counterintuitive: PANGU is optimized for planets and asteroids scene simulation, and the 3D Animation toolbox can be easily integrated with the rest of the MATLAB-developed code. However, UE4 offers complete and direct control over the scenario and the cinematography, thus allowing more precise experimental operations. Moreover, within the context of this application UE4 is the 
only software of the three which is completely free. Thus, using it to develop tools and models promotes to the rest of the community a more accessible and flexible tool to construct, improve or expand their own models.

\section{Visual Target and Lander}

As previously mentioned, the visual model representing Bennu was constructed starting from a CAD model. This was done so as to not propagate in the navigation pipeline a distribution of features reconstructed from a mathematical model, but, rather, a real one. The CAD was retrieved from the material provided by Osiris-Rex teams (NASA-GoddardUniversity of Arizona) on the mission website. In particular, the file used was the .STL of the model with a resolution of 75 centimeters [26].

It was deemed superfluous to model the background stars, as within the scope of this work, given the closeness to the surface, the magnitude of the target dominates the visual field, masking all the other, faint, objects.

Lighting, however, plays an important role in this simulation. Since the Sun can be considered a light source at infinity, it can represented as a bundle of parallel light beams, and modeled within the simulation as a Directional Light.

The direction of this light will be chosen parallel to the $X^{U E}$ axis - the $X$ axis defined in the Unreal Engine reference frame, and originating at $-\infty$. Therefore the terminator will be oriented along $Y^{U E}$, with the sunlit area belonging to the negative $X$ region. The light intensity, will be selected, heuristically, concurrently with some characteristics of the asteroid surface, trying to keep the target chromatically and visually similar to the one observed in pictures. Lastly, to obtain the colour of the light, the concept of colour temperature, mapping the temperature of a black-body expressed in $K$ into the RGB colour space, will be employed. The effective temperature of the Sun $5780 K$ [27]. A table listing $\mathrm{K}$ to RGB conversions [28] lists the hex value \#fff0e9 as the colour associated to $5800 \mathrm{~K}$, the temperature in the table which better approximates the Sun's $5780 \mathrm{~K}$. By representing \#fff0e9 in RGB we obtain the triplet $(255,240$, 233), shown in Fig. 4.

At the moment there does not seem to exist a comprehensive package validated by the community mapping surface properties. Therefore, they will be treated as a set of smaller heuristic problems.

Let us start from defining the surface radiative properties. From [19] it can be observed that the geometric albedo of Bennu is extremely low, having a value of $4.4 \%(0.04)$. Therefore, the surface will be modelled as a matte material (Roughness factor $=1$ ), completely lacking specular reflections (Specular factor $=0$ ). The albedo value, moreover, offers an important information to heuristically constraint the colour. The value of 0.04 is in fact roughly that of fresh asphalt [29]. This suggests a very low grayscale value, which was selected as $1 / 16$ of the 8 -bit scale. This is the RGB value of $(15,15,15)$, corresponding to a normalized RGB triplet of $(0.058,0.058,0.058)$, which can be observed in Fig. 5 . Ultimately, the visual result is as seen in Fig. 6.

The landmarks will require a similar process to be visually characterized. In particular, they will be assumed to be expandable structures with an aluminium coating. From an online colour repository [30] it was possible to retrieve a RGB value associated to Aluminium, equal to $(214,214,214)$, which, normalised, results in $(0.839,0.839,0.839)$, seen in Fig. 7. This material will be characterized as metallic (Metallic factor $=1$ ) with Specular and Roughness factors $=0.5$.

Fig. 4. The colour chosen for Sun illumination - RGB $(255,240,233)$

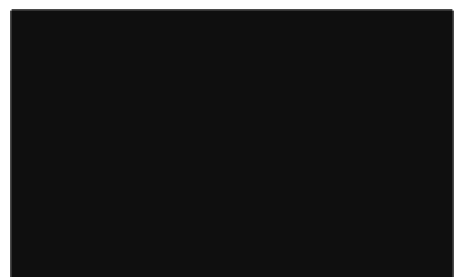

Fig. 5. The colour chosen for the asteroid surface RGB $(15,15,15)$

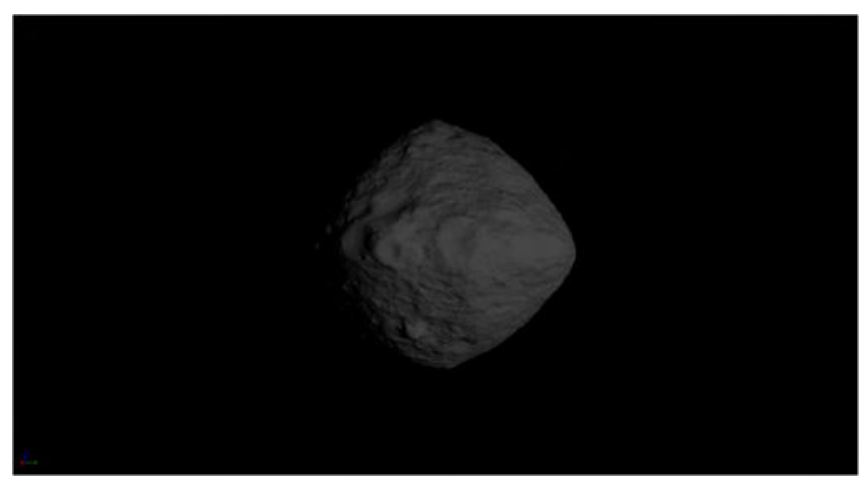

Fig. 6. The look of the asteroid in the simulation

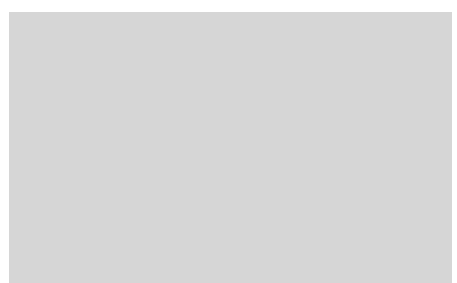

Fig 7. The colour chosen for the landmarks - RGB $(214,214,214)$

This infrastructure approach is believed to be particularly relevant for CubeSats for two main reasons. The first argument is that non-algorithmic optimizers would have a much more profound impact on low-budget objects, capable of reaching the desired accuracy only through additional assistive technology. The second argument is that a deploying mechanism for CubeSat could be reused for features, if their size has a comparable order of magnitude: therefore the technological complexity of the mission is not excessively increased. 
A lower bound to the dimension of these object within the simulation is, however, provided by the model resolution, which, again, is $0.75 \mathrm{~m}$ in this case. This because it is of interest that a landmark always correlates to structure rather than noise.

Concerning the landmarks' position, Çelik et al. [31] have investigated, albeit for a 3 body system, the deployment accuracy for passive ballistic landings on small asteroids. In that study the same landing conditions (vertical, equatorial) and backpropagation method led to a "butterfly"-like pattern, characterized by a central dispersion ellipse and two slightly asymmetric "wings". The region covered by this pattern is roughly of the same order of magnitude of the asteroid's radius itself. In particular, this field stretches for $120^{\circ}$ along the longitudinal axis and $20^{\circ}$ along the latitude extension. This distribution is approximately centered on the nominal landing position, with the spread along the latitude increasing with an increase in longitudinal distance. As the values are generally comparable in both instances these angular diameters will be imported to simulate a landing distribution.

The characterization process of the selected position for the landmarks is thoroughly characterized in Appendix A. In short however, considering the worst case and thus tignoring the central build-up, landing spots for markers are constrained to lie in one of the "wings", approximated as isosceles triangles with angular size equivalent to the one mentioned above. 10 random markers positions have been extracted from equivalent planar triangles and then projected on the surface, obtaining the results seen in Fig. 8.

The simulated sensor will be a camera with a focal length of $f=9.6 \mathrm{~mm}$. The simulated sensing plane will be constructed to be equivalent to a $1240 \times 1080$ one (1.3 MPix), with square pixels with a $5.3 \mu \mathrm{m}$ side. Therefore, the sensor height will be set to $h=6.05 \mathrm{~mm}$ and the sensor width to $w=6.94 \mathrm{~mm}$. The effective pixel number of the generated images is, however, $664 \times 578$ Pix. The above values have been modeled, with some slight difference, upon the C3D CubeSat Camera, which has already flown on CubeSat missions [32].

From all these data it is possible to define a vertical and a horizontal FOV, respectively of

$$
\begin{aligned}
& h F O V=2 \arctan \left(\frac{w}{2 f}\right)=39.75^{\circ} \\
& v F O V=2 \arctan \left(\frac{h}{2 f}\right)=34.98^{\circ}
\end{aligned}
$$

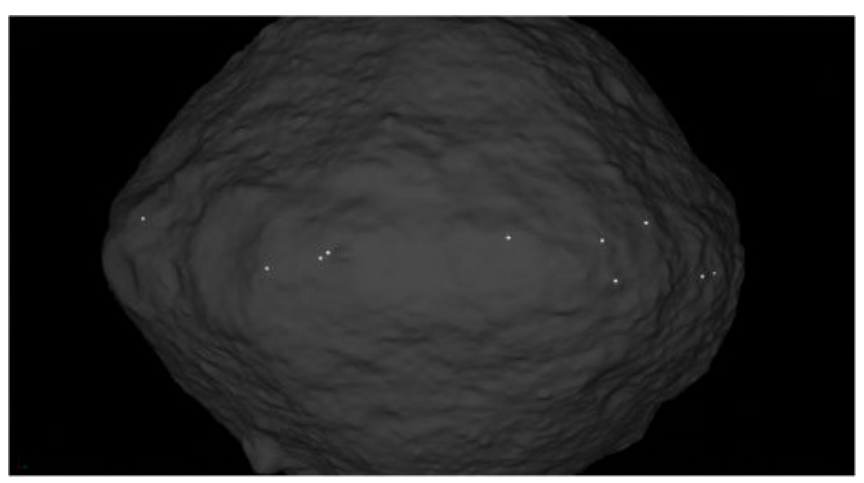

Fig. 8. The landmarks $(l=1.50 \mathrm{~m})$ as they appear on the asteroid.

\section{LANDMARK DESIGN}

The design process of the landmarks is mainly driven by mediating between two competing constraints: optical reliability and budget constraints. The former is the factor of merit in improving navigational performance and pushes for larger feature size; the latter takes into account the additional load on the mass and the volume budget by the features, and calls for smaller dimensions.

The scope of optical reliability is to provide consistent navigational information. It can be broken down into a number of secondary requirements. In particular:

- $\quad$ Restricted symmetry: this is important so as to avoid ambiguity. A certain degree of symmetry is, however, desirable as it reduces the need for active control to handle the sub-optimality or ineffectiveness of some configuration of an asymmetrical object. Nevertheless, neglecting for now the presence of surface patterns, as the degree of symmetry of an object increases, keeping its volume constant, the length of its sides (features in themselves) generally decreases, generating ambiguity;

- High visibility: this can be achieved both in active and passive ways. The former requires mechanisms capable of generating light, or changing the colour or shape of the landmark in reaction to environmental effects reducing visibility; the latter is achieved through robust design. High visibility does not necessarily equal reflectivity: for example, on a pale-tinted asteroid a VANTAblack [33] feature might be the best solution;

- $\quad$ Stability to displacing perturbations: to be effective as a navigational feature, the landmarks have to move as little as possible with respect to the surface when perturbed. The parameters controlling this are the contact surface at a given instant and the distance between the center of mass and the edge of said surface;

- Convexity: these shapes are helpful because they both reduce the amount of dark, shadowed, zones and avoid the accumulation of surface material which could "camouflage" the landmark;

- Shape Retention: it is not desirable to have features affected by the impact with an unpredictable change in their appearance, as this would lead template matching or scale reconstruction algorithms to failure.

Budget constraints require more advanced solutions to be properly addressed: for example the expandable structures mentioned in Section II. It is however out of the scope of this paper to discuss them.

Aside from having been used at a different range and for a completely different scope, Hayabusas' target markers deviate from the above conditions because they do not seem to possess easily observable sides or features. Therefore, while at the distance at which they are used they could be employed for optical scale reconstruction, their gibbous globe-like form might generate ambiguity in this process. Cubes, on the other hand, satisfy all the above mentioned constraints. Being Bennu a B Tholen type asteroid [19] to achieve high-visibility reflectivity is to be preferred to patterning or colouring. Therefore, the simulated features will be highly reflective cubes. The can be observed in Fig. 9 and Fig. 10. 


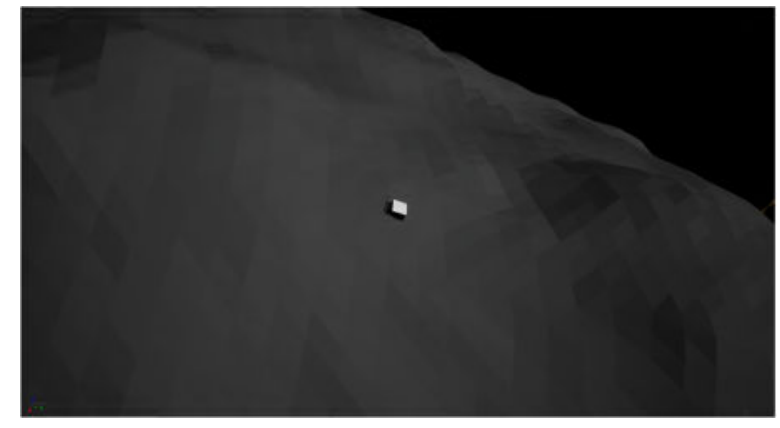

Fig. 9. A $1.5 m$ cube on the surface

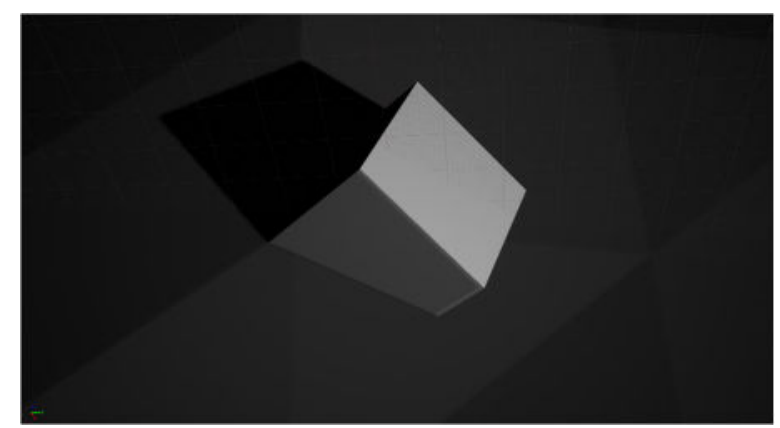

Fig. 10. Close-up of cube on the surface with its shadow. For this example, the light has been directed so as not to hit any face along its normal.

\section{RESUltS}

It is now worth noting that the reference frame defined within MATLAB differs from the one defined within Unreal Engine: namely, whereas the former is right handed, the latter is left handed. The discussion below refers to quantities constructed to be used in Unreal Engine, therefore implicitly refers to a LH system.

The landing condition of interest is a vertical, equatorial landing. The first set of parameters of the landing state to be constrained is the desired landing position of the probe, let it be $\underline{S}^{L}=\left(S_{x}^{L}, S_{y}^{L}, S_{z}^{L}\right)$. Since $\underline{S}^{L}$ lies on the equator, it is possible to write $S_{z}^{L}=0$.

Being both $S_{x}^{L}$ and $S_{y}^{L}$ belonging to the equatorial ellipse, i.e. the intersection of the equatorial plane and the ellipsoid, it is not possible to set them independently. In other words, there is only one Degree of Freedom left. This DoF can be interpreted as the angle, let it be $\theta$, of an ellipse in a classic parametric form.

As shown in Fig. 11, since the directional light is coming from the $-X$ direction, the terminator is aligned with the $Y$ axis. So as to observe the whole sunlit surface, an angle of $\theta=$ $180^{\circ}$ was chosen.

In these conditions the problem ultimately reduces itself to a one-dimensional motion, fully described by the behaviour along the $X$-axis. The equations provided, however, are not derived through this assumption, and can therefore be used for a more general case.

Ultimately

$$
\left\{\begin{array}{c}
S_{x}^{L}=R_{x} \cos (\theta)=-259 \mathrm{~m} \\
S_{y}^{L}=R_{y} \sin (\theta)=0 \mathrm{~m}
\end{array}\right.
$$

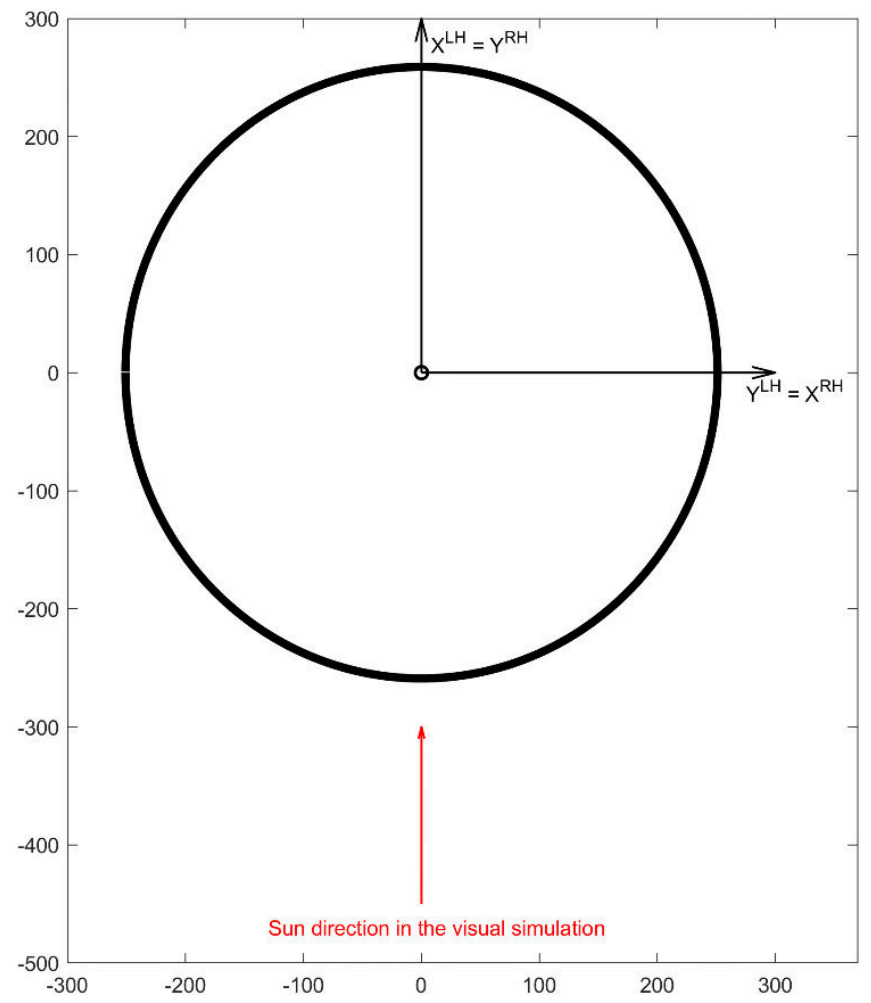

Fig. 11. The sun direction as defined within the visual simulation

Leading to $\underline{\boldsymbol{S}}^{L}=(-259,0,0)$.

To complete the landing state, the landing velocity has to be selected. This will be characterized by a direction and a magnitude - the speed. To perform a vertical landing the velocity vector has to be aligned with the local normal, which has a general form

$$
\underline{\boldsymbol{n}}(x, y, z)=\left(\frac{2 x}{R_{x}^{2}}, \frac{2 y}{R_{y}^{2}}, \frac{2 z}{R_{z}^{2}}\right)
$$

And be oriented in the opposite sense.

Which, with the data presented before, the local normal has components

$$
\begin{aligned}
& \underline{\boldsymbol{n}}\left(Q_{x}^{L}, Q_{y}^{L}, Q_{z}^{L}=0\right)=\left(\frac{2 R_{x} \cos (\theta)}{R_{x}^{2}}, \frac{2 R_{y} \sin (\theta)}{R_{y}^{2}}, 0\right) \\
& \underline{\boldsymbol{n}}\left(Q_{x}^{L}, Q_{y}^{L}, Q_{z}^{L}=0\right)=\left(\frac{2 \cos (\theta)}{R_{x}}, \frac{2 \sin (\theta)}{R_{y}}, 0\right)
\end{aligned}
$$

Thus $\underline{\boldsymbol{n}}=(-0.008,0,0)$ is obtained. Normalized with respect to its norm, $\underline{\boldsymbol{n}}$ becomes, trivially, $\underline{\hat{\boldsymbol{n}}}=(-1,0,0)$. Changing sign so as to be moving towards the target, ultimately $\underline{\boldsymbol{n}}^{L}=(0.008,0,0)$ and $\underline{\widehat{\boldsymbol{n}}}^{L}=(1,0,0)$ are obtained.

At this point, the speed might still seem a completely open parameter. However, as noted before, $V_{c}^{L}$ must not be exceeded if the probe has to stay on the surface. Thus a reduction factor $\beta$ is added so as to not operate with $V^{L}$ values too close to the critical ones, and $V^{L}=\beta V^{L}, \beta \in$ $\mathbb{R}^{+}, \beta<1$. Additionally, it has been observed that the more time a trajectory takes to bring the probe to its designated target, the more the error committed by the on board estimations grows. Ultimately, $\beta=0.67$ - roughly (2/3) seems a good compromise between estimation stability and the presence of a safety margin, therefore the selected landing speed is $V_{L}=(0.67) V_{L}^{c}$. 
With reference to Eq. 10, we can see that using the values of $R_{M}=282.36 \mathrm{~m}$ [19] (the equatorial semi-extension for the asteroid - its largest dimension), $m=7.329 \times 10^{10} \mathrm{~kg}$ retrieved from [19], and $\epsilon_{\text {crit }}=0.6$ [34]

$$
V_{L}^{c}=\sqrt{\frac{2 G m}{\epsilon_{c r i t}^{2} R_{M}}}=\sqrt{\frac{2 \times\left(6.67 \times 10^{-11}\right) \times 7.33 \times 10^{10}}{(0.6)^{2} \times 282.36}}
$$

Performing all the operations it is possible to find that $V_{L}^{c}=0.310 \mathrm{~ms}^{-1}$. Therefore $V^{L}=0.67(0.310)=$ $0.208 \mathrm{~ms}^{-1}$.

$$
\underline{\boldsymbol{V}}^{L}=V^{L} \underline{\hat{\boldsymbol{n}}}^{L}=(0.208,0,0)
$$

Hence the landing state is:

$$
\left\{\begin{array}{c}
S_{x}^{L}=-259 m \\
S_{y}^{L}=0 m \\
S_{z}^{L}=0 m \\
V_{x}^{L}=0.208 \mathrm{~ms}^{-1} \\
V_{\boldsymbol{y}}^{L}=0 \mathrm{~ms}^{-1} \\
V_{z}^{L}=0 \mathrm{~ms}^{-1}
\end{array}\right.
$$

These conditions lead to an extremely slow descent: it takes roughly 8 hours to move from about 3 kilometers away from the center of the asteroid to its surface.

The motion of the capsule will be followed from $\|\underline{r}\|=$ $2021.75 \mathrm{~m}$ to $\|\underline{r}\|=1107.40 \mathrm{~m}$, for a total time, $t^{T}$, of, roughly, 2 hours and 35 minutes $\left(t^{T}=9257 \mathrm{~s}\right)$. This segment of the trajectory has been highlighted in Fig. 12. The time step between two contiguous acquisitions is, therefore, $\delta t=1028 s=t^{T} /($ Steps -1$)$. Every image $I$ in the discrete sequence is uniquely identified by a natural number, $k$, and indicated as $I^{k}$. As a total of 10 images will be acquired through the sequence, the indexes will range from $I^{1}$ to $I^{10}$, with $I^{1}$ being the image acquired in the furthest position.

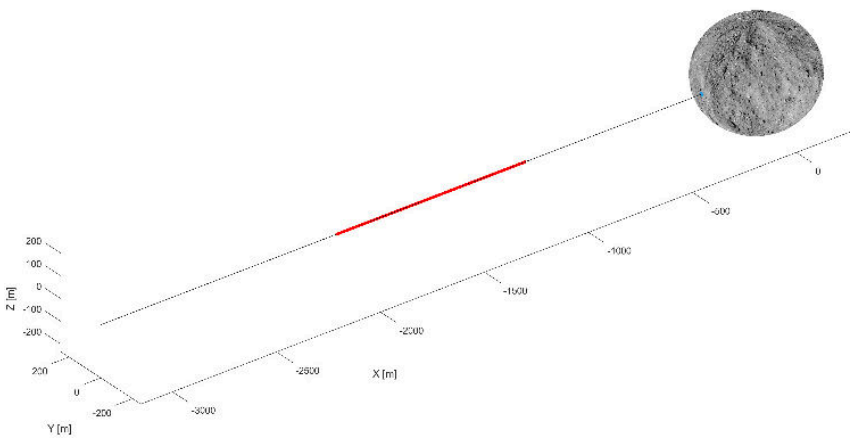

Fig. 12. The portion of the trajectory highlighted in red is the one over which VO has been performed.

Coherently with what was said before, cubes of two sizes, limited at $l=1.50 \mathrm{~m}$, will be tested: respectively, cubes with a $l=1.00 \mathrm{~m}$ side (test condition " $s \bullet$ ") and cubes with a $l=$ $1.50 \mathrm{~m}$ side (test condition " $\boldsymbol{\bullet} \bullet$ "). The amount of landmarks employed during these simulations will be 4 in one characterization, and 6 in another. To this, a condition with 0 cubes will be added, as a form of control experiment. Ultimately, this leads to 5 possible combinations of conditions to be tested, namely $T C=\{S 0, s 4, S 4, s 6, S 6\}$. It is worth noting that albeit experiments with $l=1.50 \mathrm{~m}$ might not be realistic, they provide important information to understand the effect of the cubes on navigation.

The Visual Odometry part has been performed using a standard implementation [35]. When the images are fed to the processor, Harris corners [36] are detected and matched over subsequent images. Homologous points in consecutive images are then used to construct the fundamental matrix [37] through the normalized eight-point algorithm [37]. The robustness of this process is further increased by introducing a RANSAC (RANdom SAmple Consensus) routine, so as to remove irregular points. Lastly, the fundamental matrix is used to estimate the unscaled relative translation (and rotation) between the two views. These are left as they are, and not refined through neither a local nor a global optimizer. This process, iterated sequentially, provides an estimation of the complete trajectory of the platform. It is worth noting that introducing the RANSAC makes the process nondeterministic.

The output of each step of this process is, within the scope of this work, a unit vector representing the direction of the estimated displacement, and a rotation matrix. This is because the scaling problem has been for now neglected. As noted before, the simulated motion profile is one-dimensional, evolving only along the $X$ axis. Hence, a perfect estimation from the time-step $k$ to the time-step $k+j$ would return a displacement only along $X$, with a value equal to $(k+j)-$ $k=j$. For an example of a global result, see Fig. 13 .

This means that in any estimation within this model and reference frame, components along the $Y$ axis and the $Z$ axis are entirely spurious. Hence, estimation errors in translation can be associated to these components: if they are present, the estimator is not behaving as it should. Moreover, non-roll rotations propagate on the same components when constructing the global estimation, which can therefore be also seen as indicators of rotational errors. From these considerations, it is possible to define an error metric, named $\Delta_{e}^{S}$, and characterized as (see Appendix B for more details)

$$
\Delta_{e}^{s}=(e-s)-X_{e}^{\text {Global }}
$$

Where $e$ and $s$ are the indices of the first and the last image of the sequence taken into account, and $X_{e}^{\text {Global }}$ is the cumulative $X$ value of the sequence, with the zero placed in the $I^{S}$ condition.

$\sqrt{\Delta_{e}^{S}}$ is an indicator of the effective error components; therefore normalizing $\sqrt{\Delta_{e}^{s}}$ by $(e-s)$ would return the percentage uncertainty in estimation over the trajectory of interest.

Assuming implicitly $s=1$, the values $\Delta_{5}(e=5)$ and $\Delta_{10}(e=10)$ will be presented for each case. Since, as already observed, the process contains source of randomness, it would be misleading to simply present the outputs for one instance: rather, statistical descriptors must be introduced. Namely these will be the mean, the average and the standard deviation of these parameters, computed over populations containing 300 samples. The results for $\{S 0, s 4, S 4, s 6\}$ are shown respectively, in Table I and Table II. Additionally, these values will be ordered in ascending order and plotted in Fig. 14 and Fig. 15. 


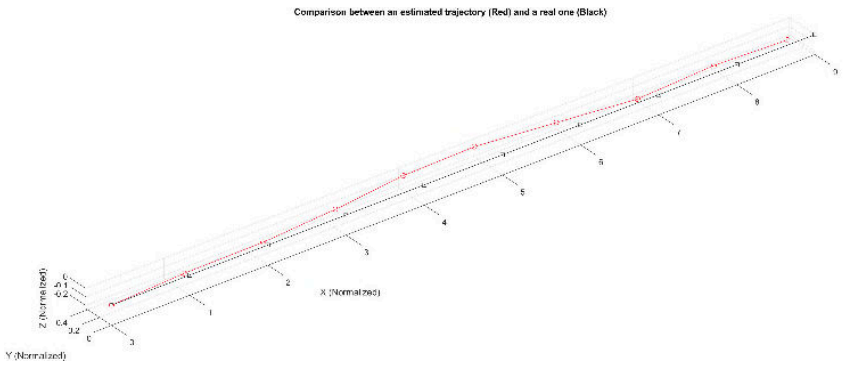

Fig. 13. Comparison between an estimated trajectory (Red) and a real one (Black)

TABLE I.

\begin{tabular}{|l|c|c|c|}
\hline \multirow{2}{*}{$\begin{array}{c}\text { Test } \\
\text { Case }\end{array}$} & \multicolumn{3}{|c|}{$\Delta_{\mathbf{5}}$} \\
\cline { 2 - 4 } & Mean & Median & Standard Deviation \\
\hline S0 & 0.3235 & 0.0467 & 0.5317 \\
\hline s4 & 0.2531 & 0.0527 & 0.4388 \\
\hline S4 & 0.1769 & 0.0285 & 0.3686 \\
\hline s6 & 0.2530 & 0.0351 & 0.4572 \\
\hline
\end{tabular}

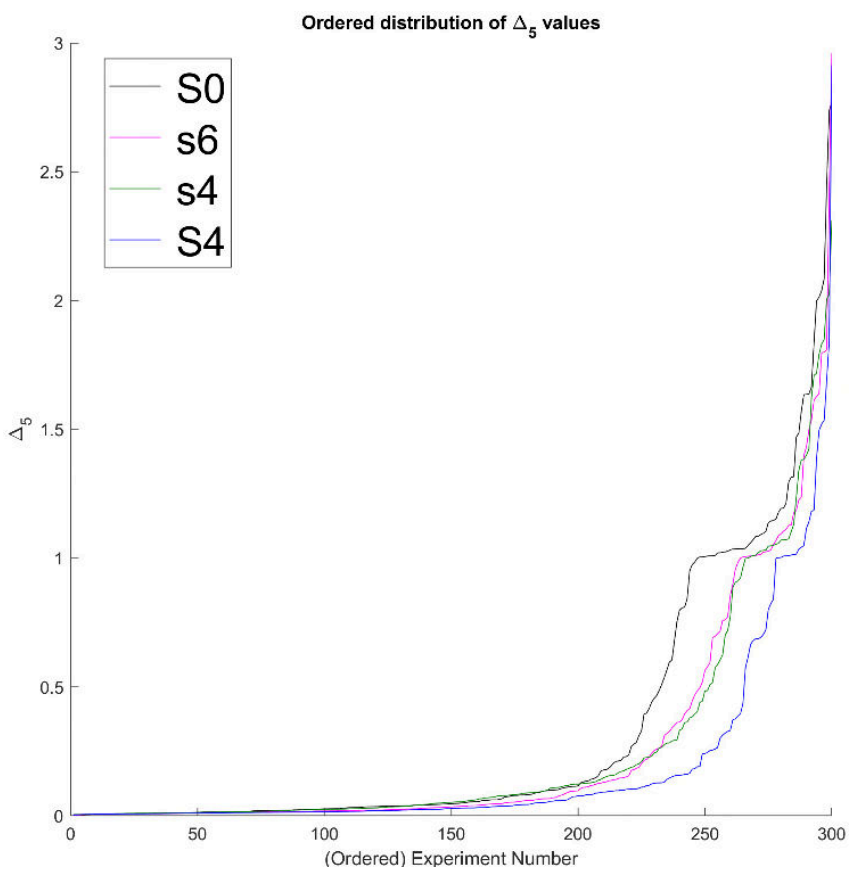

Fig. 14. Ordered distribution of $\Delta_{5}$ values

TABLE II.

\begin{tabular}{|l|c|c|c|}
\hline \multirow{2}{*}{$\begin{array}{c}\text { Test } \\
\text { Case }\end{array}$} & \multicolumn{3}{|c|}{$\Delta_{\mathbf{1 0}}$} \\
\cline { 2 - 4 } & Mean & Median & Standard Deviation \\
\hline S0 & 0.5564 & 0.1529 & 0.7179 \\
\hline s4 & 0.5878 & 0.2685 & 0.6597 \\
\hline S4 & 0.3970 & 0.1125 & 0.5524 \\
\hline s6 & 0.5726 & 0.2106 & 0.6359 \\
\hline
\end{tabular}

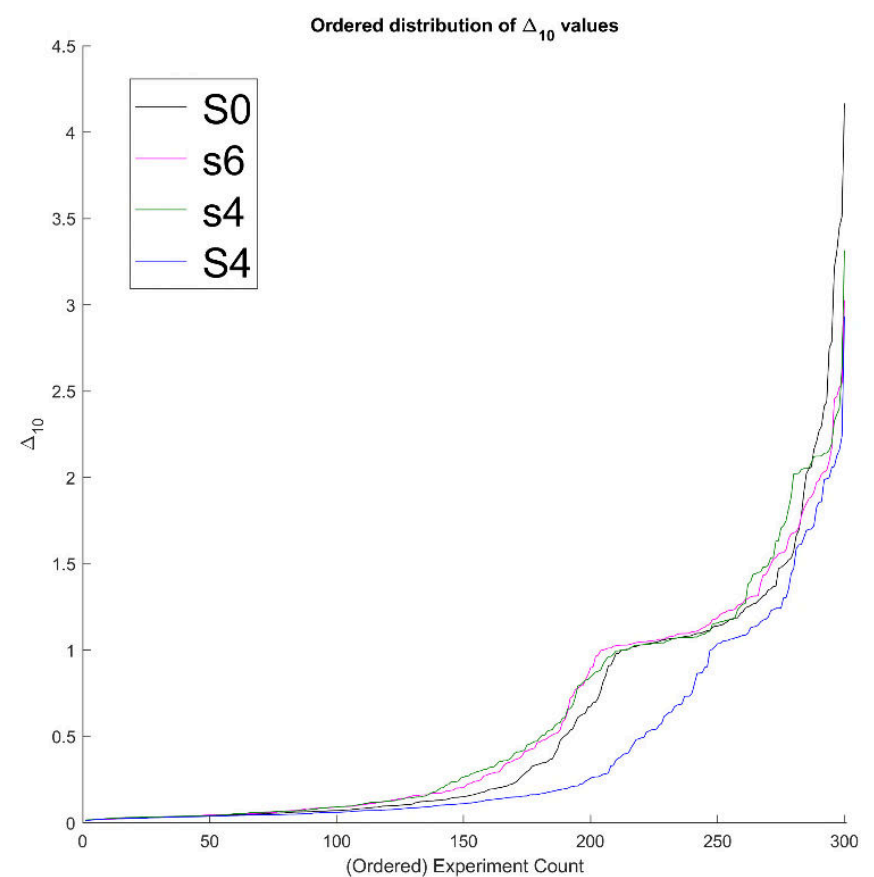

Fig. 15. Ordered distribution of $\Delta_{10}$ values

The nontrivial shape of the curve with a sharp step around a $\Delta_{5}, \Delta_{10}=1$ value can be explained by analyzing the various errors affecting the estimation. The most common type of large scale errors for this process appears to be not estimating at all a translation along the $X$ axis. A single missed estimation of this type induces a unitary $\Delta$ error; these, developing over multiple tests form the observed plateau. Therefore, the extension of this region roughly indicates the likelihood of a single non-detection event to happen. Its onset, instead, relates to the general probability of this phenomenon happening at least once.

From the above figures the consistency of this profile, and a comparable distribution of extreme values, can be observed. This means that the algorithm is behaving consistently in every test case. However, the various curves do not collapse to a single profile, but, rather, present the same shape translated horizontally. The translation is induced by the increase or the reduction of the number of test cases achieving at least an error of $\Delta$. The more results have a higher error, the more the curve appears to the left. Thus the position along the abscissae of the first onset of an exponential behavior is a good indicator of the performance of the algorithm. In other words, the more a curve is to the right, the better the algorithm is performing.

As it can be inferred from both the tables and the curves, the $S 4$ landmarks provide the best improvement from the $S 0$ case, both for $\Delta_{5}$ and $\Delta_{10}$. On the other hand, $s 4$ and $s 6$ appear to induce an identical improvement for $\Delta_{5}$, halfway between the $S 0$ and the $S 4$ test case, but then present a worse performance than $S 0$ for the $\Delta_{10}$ case.

The test case S6 has not been shown above because using the algorithm with the same parameters, would, inevitably, make the program unable to operate, and lead to crashes. This most likely happens because the stark grayscale difference between the markers and the background, coupled with a higher number of them, skews the Harris corners quality distribution. This, in turn, implies that for a broad range of min. quality values, there are fewer features having a quality 
above the acceptance threshold. In turn, this leads the cardinality of the features to be tracked is critically low, close to the minimum amount of points necessary as inputs to the algorithm. If this is coupled with a, for any reason, perceptionally troublesome points distribution, ultimately not enough features are able to be matched between consecutive views to estimate the fundamental matrix. This problem, however, might be addressed using a controller operating on the required features quality.

Let us for now, however, manually reduce the minimum quality parameter from $10^{-4}$ to $10^{-5}$. This means that the lowest score for a feature to be accepted by the algorithm is at most $10^{-5}$ of the best feature's score. This case will be defined $\mathrm{S6}^{-}$and compared to $\mathrm{SO}^{-}$and $\mathrm{S4}^{-}$. These are, respectively, $S 0$ and $S 4$ with the same reduced value of minimum feature quality. The results are presented in Table 3 and 4 and figures 18 and 19.

To more easily bridge the two sets of results, figure 16 and 17 show how the $S 0^{-}$and $S 4^{-}$set perform, respectively, against $S 0$ and $S 4$.

While implicitly written in the tables, it is worth observing how reducing the min. quality parameters improves the $\Delta_{5}$ behaviour of the marker-less case to a $s 4 / s 6$ level. This advantage, however, is entirely lost in the $\Delta_{10}$ case.

In general, what can be observed from these tests is that using lower quality features greatly reduces the effect of NAV-Landmarks on navigation performances.

TABLE III.

\begin{tabular}{|l|c|c|c|}
\hline \multirow{2}{*}{$\begin{array}{c}\text { Test } \\
\text { Case }\end{array}$} & \multicolumn{3}{|c|}{$\Delta_{\mathbf{5}}$} \\
\cline { 2 - 4 } & Mean & Median & Standard Deviation \\
\hline S0 $^{-}$ & 0.2649 & 0.0452 & 0.4387 \\
\hline S4 $^{-}$ & 0.2362 & 0.0344 & 0.4766 \\
\hline S6 $^{-}$ & 0.2626 & 0.0412 & 0.4738 \\
\hline
\end{tabular}

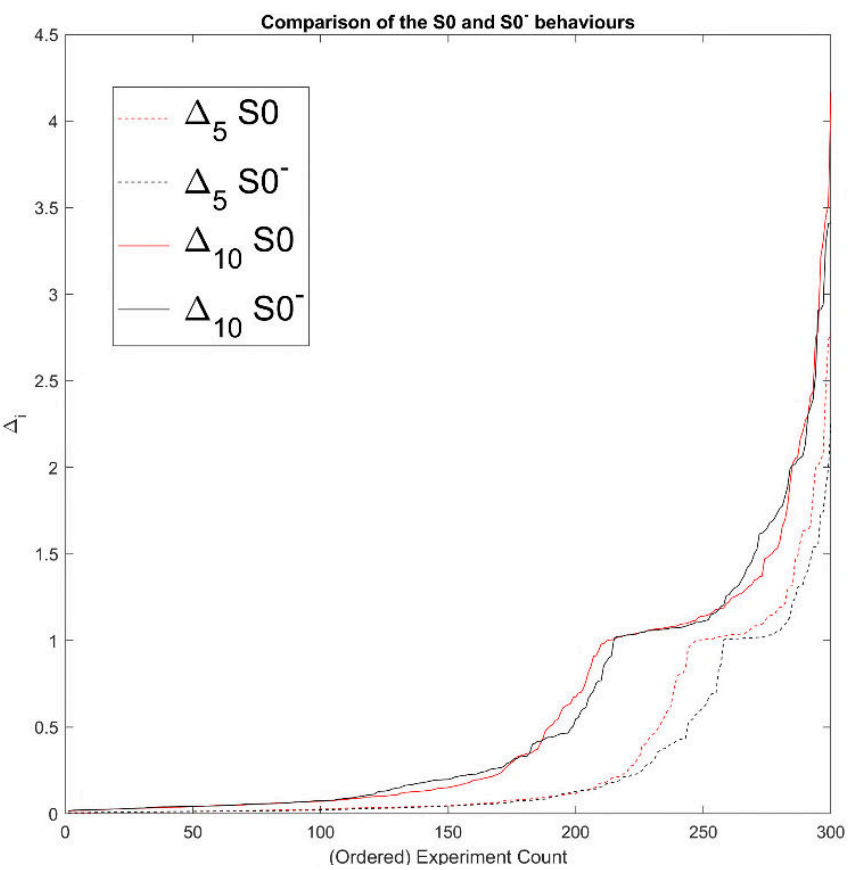

Fig. 16. Comparison of $S 0$ and $S 0^{-}$

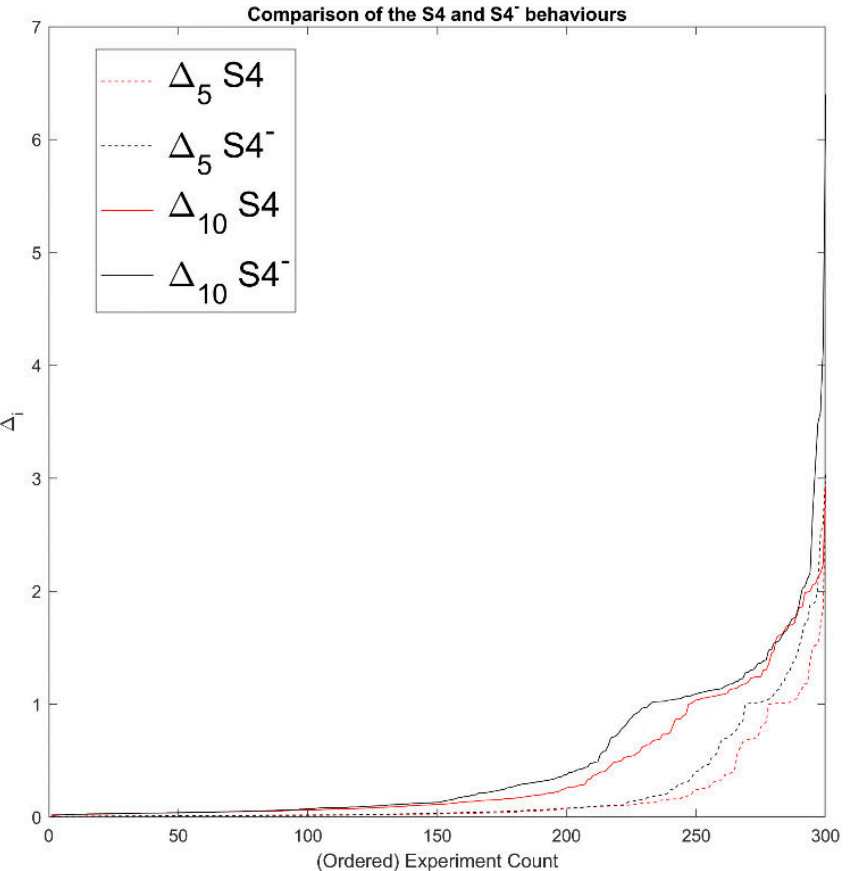

Fig. 17. Comparison of $S 4$ and $S 4^{-}$

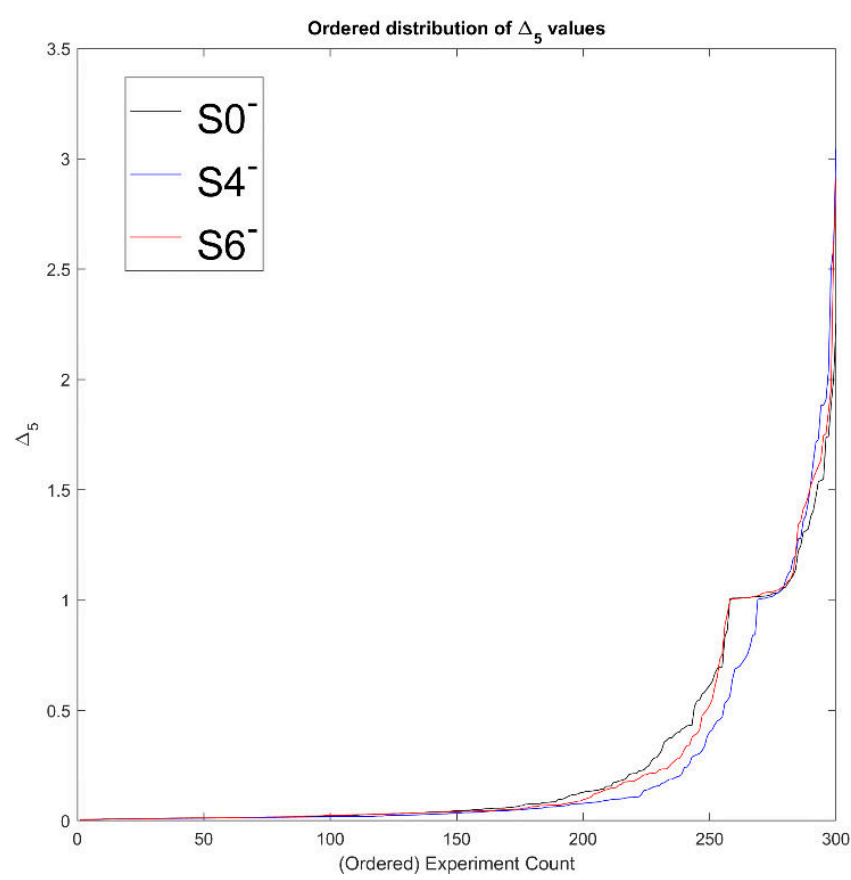

Fig. 18. Ordered distribution of $\Delta_{5}$ values

TABLE IV.

\begin{tabular}{|l|c|c|c|}
\hline \multirow{2}{*}{$\begin{array}{c}\text { Test } \\
\text { Case }\end{array}$} & \multicolumn{3}{|c|}{$\Delta_{\mathbf{1 0}}$} \\
\cline { 2 - 4 } & Mean & Median & Standard Deviation \\
\hline S0 $^{-}$ & 0.5555 & 0.1997 & 0.6979 \\
\hline S4- $^{-}$ & 0.4925 & 0.1305 & 0.7305 \\
\hline S6- $^{-}$ & 0.4832 & 0.1397 & 0.6488 \\
\hline
\end{tabular}




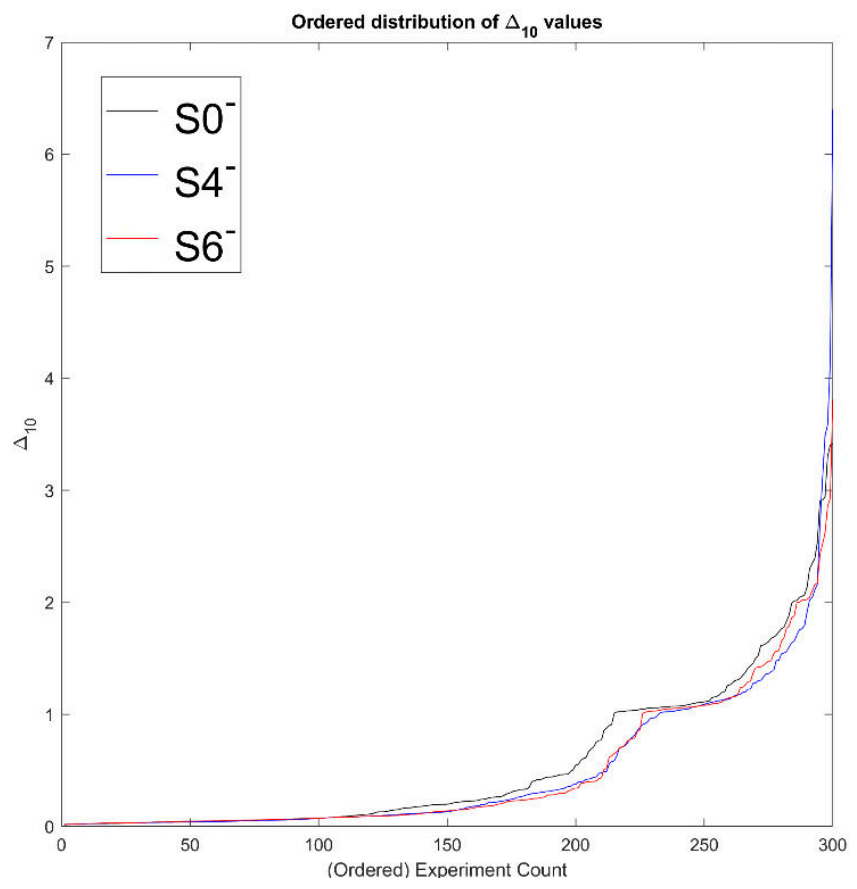

Fig. 19. Ordered distribution of $\Delta_{10}$ values

\section{Conclusions}

Landing on the surface of distant celestial bodies requires an elevated degree of autonomy. Augmenting the environment to a cooperative state might enable additional, more complex operations even for probes with limited on-board capabilities, like CubeSats.

In particular, within the scope of this paper, this idea has been explored by analyzing the effect of the introduction of artificial visual landmarks on the surface of a small asteroid on the visual odometry performance of the landing probe. The probe is at a medium-far range, and employs only a visible camera to navigate. In this regard, this study can be intended also as a pilot work in visual semiotics for space robots.

This has been done by developing a simulation package coupling the dynamics and the visual aspect of the problem. The visual portion has accommodated the introduction of the markers on the surface of an asteroid model. The dynamics part has been pivotal in generating a trajectory associated to a precise landing spot, and in retrieving the position of the probe with a consistent temporal frequency.

The results are definitely promising, as it has been observed that the landmarks improve the unscaled results in most cases. More research on the topic is however needed to clarify and explain some unexpected behaviour that arose. Moreover, issues related to perception, as the influence of extreme values in the scene were highlighted. Future work will therefore focus on dealing with these issues, on evaluating the optimal feature detector, on trying to implement scale reconstruction, and on comparing the performance of these "physical" optimizers with computational optimizers.

\section{APPENDICES}

\section{A. DEFINING THE LANDMARKS POSITION}

Since distortions and projection issues would ultimately render prohibitive treating this problem analytically or numerically on the real Bennu surface, an approximation has been employed. The points were defined within a region of a planar rectangle with dimensions $a Y \times b Z$ centered in the point $\left(-R_{x}, 0,0\right)$ and parallel to the $Y-Z$ plane. The size in meters of both sides has been obtained using again the DEEVE approximation. In particular, $a Y$ is the chord along the equator defined by an arch having an angular diameter of $120^{\circ}$ and centered on the $X$ axis; $b Z$ is the chord defined on the meridian characterized by $Y \equiv 0$ by points having a latitude of $\pm 10^{\circ}$. Let the points defining the chord $a Y$ be $Y^{\prime}$ and $Y^{\prime \prime}$, and let the points defining the chord $b Z$ be $Z^{\prime}$ and $Z^{\prime \prime}$. Graphically, the construction of these objects is represented in Fig. 1a; Eq. 1a and 1b, on the other hand, provide a mathematical description of these objects

$$
\begin{aligned}
& a Y=Y^{\prime}-Y^{\prime \prime}=R_{y}\left(\sin \left(120^{\circ}\right)-\sin \left(240^{\circ}\right)\right)=435 m(1 \mathrm{a}) \\
& b Z=Z^{\prime}-Z^{\prime \prime}=R_{z}\left(\sin \left(10^{\circ}\right)-\sin \left(-10^{\circ}\right)\right)=81 m
\end{aligned}
$$

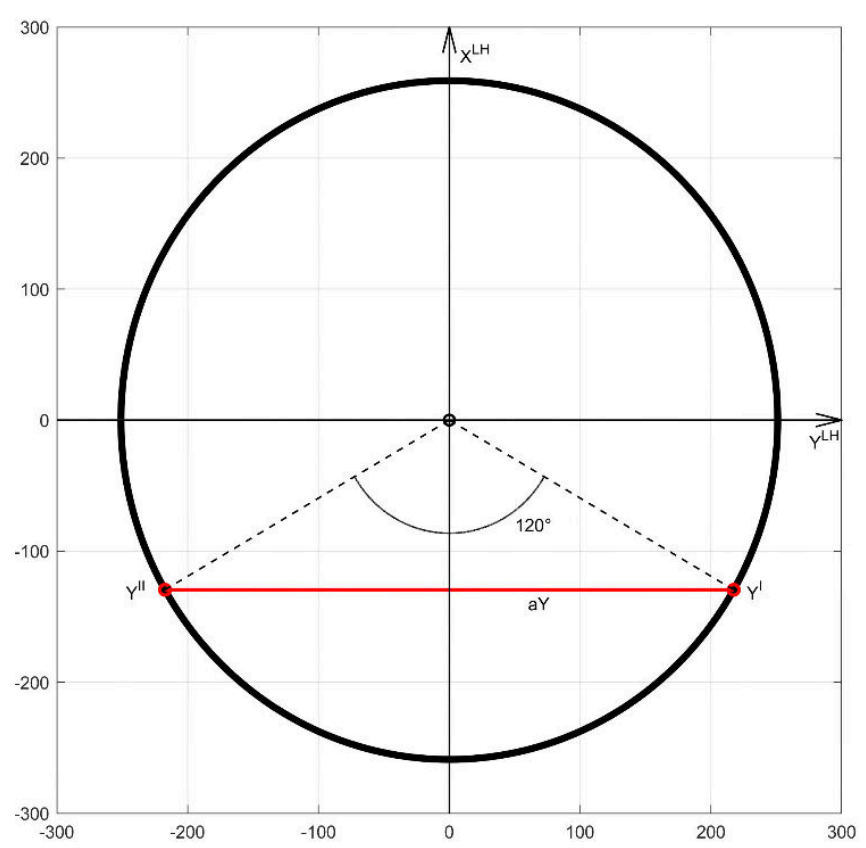

Fig. 1a. A visual definition of $a Y$

To maintain consistency with the reference frame utilized within Unreal Engine, this ellipsoid has been defined in a left handed reference frame (superscript "LH").

The initial markers parameters $(\|Y\|, \operatorname{sign}(Y)$, $\|Z\|, \operatorname{sign}(Z))^{0}$ have been be extracted from uniform distributions and accepted only and only if they would satisfy the geometric constraint of belonging to the triangles. The results obtained for the 10 extractions defining the 4 and the 6 landmarks case is shown in Fig. 2a. Subsequently, to place the landmarks on the target, the plane is translated along the $X$-axis and landmarks are introduced in the point where they first intercept the surface. 


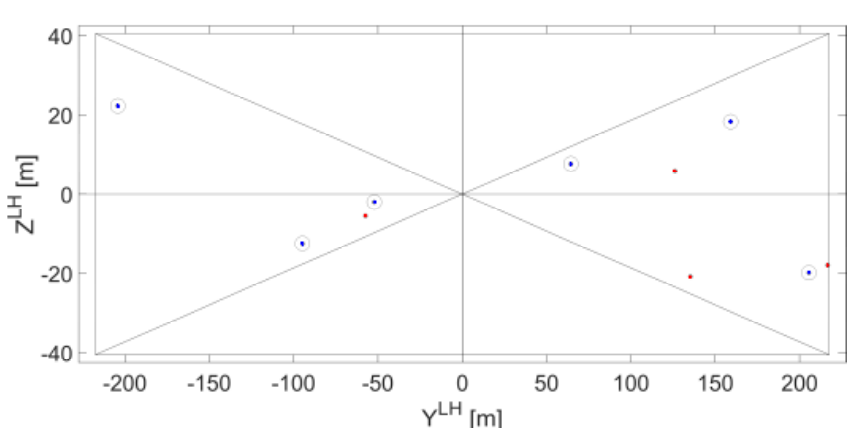

Fig 2a. The point distribution for 6 cubes (circled dots) and for 4 cubes (simple dots)

\section{B. Selecting An ERror Metric}

The output of the monocular visual odometry algorithm is a unit vector with components $\{X, Y, Z\}$ representing the estimated direction of motion.

$$
\sqrt{X^{2}+Y^{2}+Z^{2}}=1
$$

Scale can eventually be introduced at a later stage using the information provided by a secondary source.

By squaring both terms of the equation, it is possible to rewrite Eq. (1b) as

$$
X^{2}+Y^{2}+Z^{2}=1
$$

Now, let us represent this vector as the composition of two components. Namely, a transversal circle of radius $r_{t}$ placed at a longitudinal distance $X$ from the origin of the vector. Therefore, $r_{t}$ can be written as

$$
r_{t}^{2}=Y^{2}+Z^{2}=\varepsilon
$$

Being $X \in[-1 ; 1], X^{2}$ is bound to have values such that $X^{2} \in[0 ; 1]$. Therefore, being

$$
X^{2}=1-\varepsilon
$$

$\varepsilon$ has to be a non-negative quantity; specifically $\varepsilon \in$ $[0 ; 1]$. Without loss of generality, $\forall \varepsilon \in[0 ; 1]$ it is possible to find an ancillary value $\gamma \in[0 ; 1]$ such that

$$
\varepsilon=2 \gamma-\gamma^{2}
$$

Introducing Eq. (5b) in Eq. (4b) it is possible to obtain

$$
X^{2}=1+\gamma^{2}-2 \gamma=(1-\gamma)^{2}
$$
form

Taking the square root of both terms ultimately returns the

$$
|X|=|1-\gamma|
$$

Let us now define the estimation error on the longitudinal component, let it be $\delta_{K}^{x}$. The $K$-th estimation will be the one describing the motion from the $(k-1)$-th frame $\left(I^{k-1}\right)$ to the k-th frame $\left(I^{k}\right) . \delta_{K}^{x}$ will be the difference between the projection of the effective motion on the $X$-axis, $X^{e f f}$, and the $X$ component estimated by the Visual Odometry routine, $\hat{X}$

$$
\delta_{i}^{x}=X_{i}^{e f f}-\hat{X}_{i}
$$

By summing over the complete trajectory

$$
\Delta^{x}=\sum_{i=s}^{e} \delta_{i}^{x}
$$

The right side of Eq, (9b) can be expanded by distributing the sum, thus obtaining

$$
\Delta^{x}=\sum_{i=S}^{e} X_{i}^{e f f}-\sum_{i=S}^{e} \hat{X}_{i}
$$

Let us now go back to the effective motion profile. Again, given all the constraints introduced in the paper the motion reduces itself to a positive translation along the $X$ axis. This means that a perfect $\mathrm{VO}$ estimation would return, for every step

$$
X^{e f f}=(1-\gamma)_{\gamma \equiv 0}=1
$$

Leading to the simplification of the first term of the right side of Eq. (10b) to

$$
\sum_{i=s}^{E} 1=E-S+1
$$

It must now be remembered that $\delta_{i}^{x}$ is defined between two camera acquisitions, $i$ being the second of them. Therefore, if the sum necessarily begins from the displacement $i=S$, the actual first frame of the sequence is $=S-1 \Leftrightarrow S=s+1$. By rewriting Eq. (12b) in term of frames ultimately it is possible to obtain

$$
\sum_{i=s}^{E} 1=e-(s+1)+1=e-s
$$

Let us now focus on the sum containing $\gamma$. It is deemed highly unlikely, given how clear the expansion is within this study, that the $\mathrm{VO}$ algorithm would ever estimate a negative $X$ translation. Therefore, it is possible to remove the absolute value and select only the positive values of $(1-\gamma)$. By distributing also this sum it is possible to obtain

$$
\sum_{i=s}^{E}(1-\gamma)=(e-s)+\sum_{i=s}^{E}(-\gamma)
$$

By substituting Eq. (13b) and Eq. (14b) in Eq. (10b) ultimately it is proven that $\Delta^{x}$ is a function exclusively of $\gamma$

$$
\Delta^{x}=-\left(\sum_{i=s}^{E}(-\gamma)\right)
$$

Therefore, $\Delta^{x}$ depends only on a sum of functions of the erroneous components of the estimation, $Y$ and $Z$. Moreover, using the definition provided in Eq. (10b) it is possible to see that Eq. (15b) is also the $\Delta_{e}^{S}$ defined in the paper. As seen before, retrieving its value is rather straightforward, and as just shown it effectively provides a metric on the behaviour of the erroneous components. Specifically, the order of magnitude of the spurious components goes roughly as $\sqrt{\Delta^{x}}$.

\section{ACKNOWLEDGMENT}

Marco Zaccaria Di Fraia's PhD is sponsored by Thales Alenia Space.

\section{REFERENCES}

[1] Schoolcraft, Josh, Andrew Klesh, and Thomas Werne. "MarCO: interplanetary mission development on a CubeSat scale." Space Operations: Contributions from the Global Community. Springer, Cham, 2017. 221-231.

[2] Swartwout, Michael. "The first one hundred cubesats: A statistical look." Journal of Small Satellites 2.2 (2013): 213233.

[3] Martin-Mur, Tomas J., Eric D. Gustafson, and Brian T. Young. "Interplanetary CubeSat navigational challenges." (2015).

[4] Krishnakumar, Kalmanje S., et al. "A Proposed Strategy for the US to Develop and Maintain a Mainstream Capability Suite." (2012). 
[5] Oda, Mitsushige, Kouichi Kibe, and Fumio Yamagata. "ETS-VII, space robot in-orbit experiment satellite." Proceedings of IEEE international conference on robotics and automation. Vol. 1. IEEE, 1996.

[6] Hui, Cheng, et al. "Autonomous takeoff, tracking and landing of a UAV on a moving UGV using onboard monocular vision." Proceedings of the 32nd Chinese Control Conference. IEEE, 2013.

[7] Babinec, Andrej, et al. "Visual localization of mobile robot using artificial markers." Procedia Engineering 96 (2014): 1-9.

[8] dos Santos Cesar, Diego Brito, et al. "An evaluation of artificial fiducial markers in underwater environments." OCEANS 2015-Genova. IEEE, 2015.

[9] Epp, Chirold D., Edward A. Robertson, and Tye Brady. "Autonomous landing and hazard avoidance technology (ALHAT)." 2008 IEEE Aerospace Conference. IEEE, 2008. [10] Yu, Meng, Hutao Cui, and Yang Tian. "A new approach based on crater detection and matching for visual navigation in planetary landing." Advances in Space Research 53.12 (2014): 1810-1821.

[11] Escrig, Felix, and Juan Pablo Valcarcel. "Geometry of expandable space structures." International Journal of Space Structures 8.1-2 (1993): 71-84.

[12] Schenk, Mark, et al. "Review of inflatable booms for deployable space structures: packing and rigidization." Journal of Spacecraft and Rockets 51.3 (2014): 762-778.

[13] Kubota, Takashi, Tatsuaki Hashimoto, and Jun'ichiro Kawaguchi. "Motion planning of intelligent explorer for asteroid exploration mission." Motion Planning (2008): 243.

[14] Hayabusa2 Information Fact Sheet - v2.3

[15] Kubota, Takashi, et al. "Collision dynamics of a visual target marker for small-body exploration." Advanced Robotics 21.14 (2007): 1635-1651.

[16] "The Pinpoint Touchdown - TargetMarker 1 (PPTD-

TM1) operation.", JAXA Hayabusa2 project. (2019).

[Online] Available at:

http://www.hayabusa2.jaxa.jp/en/topics/20190515e_PPTD$\underline{\mathrm{TM} 1 /}$

[17] Ogawa, Naoko, Fuyuto Terui, and Jun'ichiro Kawaguchi. "Precise Landing of Space Probe on Asteroid Using Multiple Markers." IFAC Proceedings Volumes 43.15 (2010): 172-177.

[18] Lauretta, D. S., et al. "The OSIRIS - REx target asteroid (101955) Bennu: Constraints on its physical, geological, and dynamical nature from astronomical observations." Meteoritics \& Planetary Science 50.4 (2015): 834-849.

[19] Lauretta, D. S., et al. "The unexpected surface of asteroid (101955) Bennu." Nature 568.7750 (2019): 55.

[20] DellaGiustina, D. N., et al. "Properties of rubble-pile asteroid (101955) Bennu from OSIRIS-REx imaging and thermal analysis." Nature Astronomy 3.4 (2019): 341.

[21] Scheeres, Daniel J. Orbital motion in strongly perturbed environments: applications to asteroid, comet and planetary satellite orbiters. Springer, 2016.

[22] Carlson, B. C. "Computing elliptic integrals by duplication." Numerische Mathematik 33.1 (1979): 1-1

[23] "PANGU - Planet and Asteroid Natural scene Generation Utility - STAR-Dundee", STAR-Dundee.

[Online]. Available at: https://www.star-

dundee.com/products/pangu-planet-and-asteroid-naturalscene-generation-utility/
[24] "Simulink 3D Animation", Uk.mathworks.com. Available at: https://uk.mathworks.com/products/3danimation.html?requestedDomain=

[25] "Unreal Engine | What is Unreal Engine 4", Unrealengine.com. [Online]. Available at: https://www.unrealengine.com/

[26] "Bennu Shape Model Files - OSIRIS-REx Mission", OSIRIS-REx Mission, 2019. [Online]. Available at: https://www.asteroidmission.org/updated-bennu-shapemodel-3d-files/

[27] D. Rouan, "Effective Temperature," in Encyclopedia of Astrobiology, Springer Berlin Heidelberg, 2011, pp. 479480.

[28] "What color is a blackbody? - some pixel rgb values", Vendian.org. [Online]. Available at: http://www.vendian.org/mncharity/dir3/blackbody/

[29] Lambrechts, Johannes, and Saurabh Sinha. Microsensing networks for sustainable cities. Switzerland: Springer International Publishing, 2016.

[30] "Hex Colors, Color Picker, Directory, Schemes, Paint Search \& Conversions", Encycolorpedia.com. [Online]. Available at: https://encycolorpedia.com/

[31] Çelik, Onur, et al. "Reliability Analysis of Ballistic Landing in Binary Asteroid 65803 (1996 GT) Didymos under Uncertainty and GNC Error Considerations." 26th International Symposium on Spaceflight Dynamics. 2017.

[32] "C3D CUBESAT CAMERA | XCAM", Xcam.co.uk. [Online]. Available at: http://www.xcam.co.uk/c3d-cubesatcamera

[33] Y1lmaz, Ahenk. "Color of absence and presence: Reconsidering black in architecture." Color Research \& Application 42.3 (2017): 378-387.

[34] Maurel, Clara, et al. "Numerical simulations of the contact between the lander mascot and a regolith-covered surface." Advances in Space Research 62.8 (2018): 20992124.

[35] Scaramuzza, Davide, and Friedrich Fraundorfer. "Visual odometry [tutorial]." IEEE robotics \& automation magazine 18.4 (2011): 80-92.

[36] Harris, Christopher G., and Mike Stephens. "A combined corner and edge detector." Alvey vision conference. Vol. 15. No. 50. 1988.

[37] Hartley, Richard, and Andrew Zisserman. Multiple view geometry in computer vision. Cambridge university press, 2003.

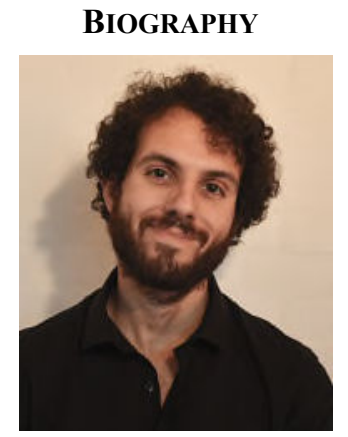

Marco Zaccaria Di Fraia is currently pursuing a PhD in Defence and Security at Cranfield University (UK). His PhD project, sponsored by Thales Alenia Space, is focused on developing robust landing solutions for autonomous spacecraft. He received his B.Sc. and M.Sc. in Aerospace Engineering from University of Naples "Federico II" (Italy) in 2015 and 2017, respectively. While pursuing his M.Sc., he spent nine months working as an intern in the Aerospace Systems Team, working on data fusion of EO sensors for pose determination of uncooperative spacecraft. His current research interests are Visual Navigation, AI, Robotics and Space Landings. 


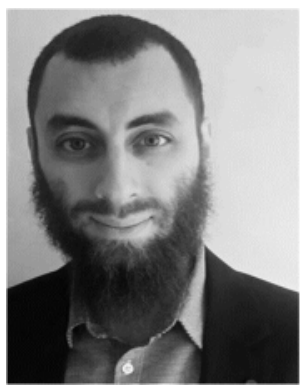

Lounis Chermak (M'12) received his Engineering Degree in Electrical Engineering from Polytech' Grenoble, Grenoble, France, in 2010 and then an MSc. and Ph.D. degrees in Computer Vision from Cranfield University, Cranfield - Shrivenham, UK, in 2011 and 2014 respectively. From 2014 to 2017 he was Research Fellow and since 2017 he is Assistant Professor in Computer Vision and Autonomous Systems at Cranfield University, Shrivenham, UK, where he currently leads the Autonomy Group and Autonomous Systems Laboratory. in the Centre of Electronic Warfare Information and Cyber, at School of Defence and Security. He has authored and co-authored several technical papers, and has been reviewer for high impact journals and conferences. His research interests includes development and implementation of techniques and algorithms for visual based aerial, ground and space applications towards autonomous mobility. He is a recipient of the Selwyn Award, from the Royal Photography Society in 2017. He has been appointed technical member and Chair of one of the Autonomous Vehicle Technology Exploratory Team at NATO Science and Technology Organization since 2018.

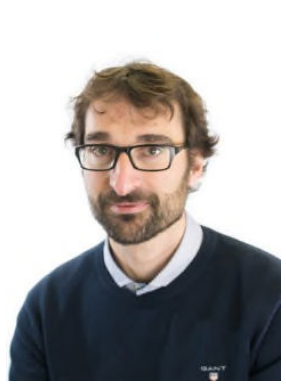

Joan Pau Sánchez is Lecturer in Space Engineering at Cranfield University since 2015. He has more than 14 years of research experience on the fields of Astrodynamics, Trajectory Design, Orbit Determination and Navigation and Space System Engineering (hindex 13 according to Scopus). Some of his recent work addresses issues with regards the trajectory design and navigation capabilities of near or mid-term space missions. In particular, he is involved in a project looking at the autonomy and the navigation capabilities of shoebox sized Cubesats, as well as he is part of the Comet Selection Team for ESA Comet Interceptor mission.

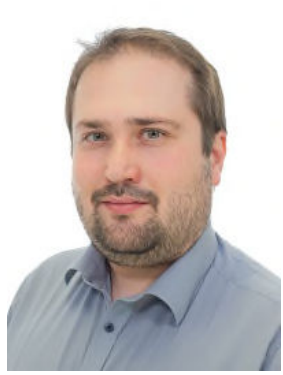

Leonard Felicetti is a Lecturer in Space Engineering in Cranfield University (UK). He obtained his Ph.D. and he was a Post-Doc Researcher in Sapienza - University of Rome (Italy). In 2015, he was Honorary Research Associate at University of Glasgow (UK) and then, Associate Senior Lecturer in On-board Space Systems in Lulea University of Technology (Sweden). He joined Cranfield University (UK) in 2019. Leonard's main research interests are on Spacecraft Formation Flying; Guidance, Navigation and Control; Spacecraft Orbital and Attitude Control; Space Robotics; Autonomous Distributed Space Systems; Spacecraft and Mission Design.

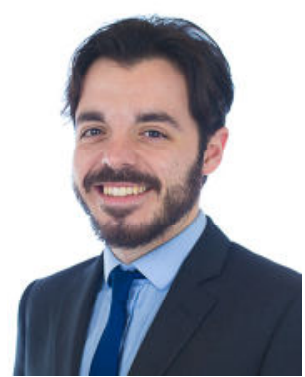

Antonio F. Scannapieco obtained his B.Sc. and M.Sc. in Aerospace Engineering with honors at University of Naples "Federico II" in 2011 and 2013, respectively. His fields of interest were aerospace remote sensing, space and autonomous systems. In 2017 Antonio was awarded his PhD in Industrial Engineering from University of Naples "Federico II". His doctoral research activities dealt with developing design strategies and processing approaches for a novel ultralight radar sensor, in order to provide miniaturized Unmanned Aircraft Systems with autonomous operations, mainly but not exclusively indoors. At the same time, he tested commercial lightweight radar to assess potentialities towards autonomous navigation, sense-and-avoid imaging. While completing his PhD, Antonio was selected as participant for Fraunhofer FHR International Summer School on Radar/SAR and for Fraunhofer IAIS TRADR Summer School on Autonomous Micro Aerial Vehicles. He was also Visiting Researcher at Fraunhofer FHR from July to December 2016 After PhD completion, Antonio Scannapieco joined Cranfield University as Research Fellow in Navigation and Guidance at Signals and Autonomy Group. His research activities mainly focused on the "H2020 I3DS"
(Integrated 3D Sensors) project, in terms of vision-based navigation for orbital and planetary activities and sensor pre-processing. He also worked for the DSTL-funded "R\&D into Non-GNSS Navigation" project, dealing with multispectral navigation. Antonio is also reviewer for several high-level journals and international conferences mainly but not exclusively in the field of aerospace engineering and he was Rapporteur in one session of IAC 2018. 
NAV-Landmarks: deployable 3D infrastructures to enable CubeSats navigation near asteroids

\author{
Di Fraia, Marco Zaccaria
}

IEEE

Di Fraia MZ, Chermak L, Cuartielles J-P, et al., (2020) "NAV-Landmarks: Deployable 3D Infrastructures to Enable CubeSats Navigation Near Asteroids," 2020 IEEE Aerospace Conference, 7-14 March 2020, Big Sky, MT, USA, pp. 1-14 https://doi.org/10.1109/AERO47225.2020.9172720

Downloaded from Cranfield Library Services E-Repository 